\title{
Low-Cost Computers for Education in Developing Countries
}

\author{
Jeffrey James
}

Accepted: 29 August 2010/Published online: 11 September 2010

(C) The Author(s) 2010. This article is published with open access at Springerlink.com

\begin{abstract}
This paper studies the distribution of computer use in a comparison between two of the most dominant suppliers of low-cost computers for education in developing countries (partly because they involve diametrically opposite ways of tackling the problem). The comparison is made in the context of an analytical framework which traces the changing characteristics of products as income rises over time. The crucial distinction turns out to be the way sharing is handled in the two cases. In the one no sharing is allowed while in the other sharing is the basis of the entire product design. Put somewhat differently, the one computer is intensive in a high-income characteristic whereas the other relies entirely on a low-income characteristic.
\end{abstract}

Keywords Appropriate technology $\cdot$ OLPC $\cdot \mathrm{N}$ Computing $\cdot$ Innovation

\section{Introduction}

In this paper I do not seek to compare all the low-cost computers on the market for providing education to schools in developing countries. ${ }^{1}$ Rather, I compare just two of the most dominant of them which also happen to constitute diametrically opposite ways of going about this task. Nor, when evaluating these alternatives do I attempt a comprehensive approach. My more limited goal is to ask which of the two competitors generates a more equal distribution of computer use and why. Which of the two in other words can be described as the more appropriate product (where appropriateness is defined to include the way in which the product is accessed)? Concretely the question is as follows: given a certain amount of income, which type of computer provides more students with access and use? This is admittedly only one element in the selection of a low-cost computer but it is surely an important one.

${ }^{1}$ The list includes refurbished, second-hand computers, Intel's 'Classmate', 'N Computing', 'OLPC' (see the InfoDev site on The World Bank page).

J. James $(\bowtie)$

Tilburg University, Tilburg, Netherlands

e-mail: m.j.james@uvt.nl 
I find that the answer to this question depends rather heavily on what might be termed 'conditions of use' (or modes of access) characteristics. Some such characteristics originate in and are based on conditions in the developing countries while others are more typical of contemporary developed countries. And partly for this reason some characteristics of computers turn out to be appropriate and others happen to be inappropriate for the majority of those living in poor countries. This historically oriented line of argument, with its emphasis on high and low-income characteristics is developed in an analytical framework which pays particular reference to the individual and shared forms of access to computers. These characteristics are emphasized because they seem best to discriminate between the two computers (although of course there are other distinctions between them as well).

The framework is based on and begins with the recognition that goods should be seen as bundles of embodied characteristics. Goods in themselves therefore cease to be objects of desire; they are desired instead because of the characteristics they embody. ${ }^{2}$ Indeed, it is only according to this non-traditional theory of demand that the distributional impact of products can properly be analyzed and understood. I begin though with a brief comparison of the two cases at hand, namely, the OLPC programme and the desktop visualization solution from the firm ' $\mathrm{N}$ Computing' (it is brief partly because I do not cover the technical difference between the products).

Notice too that I take a distinctly economics approach to the issue by focusing on costs. In so doing, however, I fully recognize that there are other important dimensions of choice that cannot (easily) be fitted into this approach. There are those for example who argue that a laptop provides a different and more comprehensive educational experience than a regular desk-top alternative (among other things, it is claimed that laptops foster more curiosity in a child and engage the wider family in his or her educational experience). Simplicity is another dimension which cannot be quantified but which should be considered in a decision to choose a type of computer.

\section{A Brief Comparison}

The goal of the OLPC (one laptop per child) programme is to ensure that every child of school-going age in the developing world is provided with his or her own laptop. Children should be able 'to engage effectively with their own personal laptop networked to the world so that they can openly learn and learn about learning' (http://www.laptop foundation.org/program/).

To this end, the (XO computer) was designed, mainly in the MIT Media Lab Cambridge, Massachusetts to produce an ultra low-cost laptop with features designed for developing rather than developed countries (such as open-source software, low power, mesh networked devices and ruggedness). An initial prototype was presented to the WSIS (World Summit on the Information Society) in 2004 and 2 years later full operations began. Although the target of the project has been to produce a $\$ 100$ laptop, this has not yet been achieved (with the current price around \$175).

Sales are made mainly to governments though a 'give one get one' campaign allows private buyers in developed countries to donate 1 computer to a school in the developing world.... Although the (XO) cannot claim to be the first well known computer for developing countries.... it can certainly be said to have spawned a lot of interest and activity in this previously neglected market.

\footnotetext{
${ }^{2}$ See Stewart (1997).
} 
Another major competitor is "N Computing", an American-based firm whose product, desktop virtualization, derives from the recognition that the average personal computer requires only $5 \%$ of its capacity (Greenberg 2008). The firm's achievement has been to devise new software that exploits the unused part of a computer's capacity. This involves the simultaneous use of a single computer by numerous children (up to 30 in fact). The basic idea is to convert the unused capacity into individual workplaces that give users the same experience as if they were working with their own computers.

Crucial to desktop virtualization is an access device. They are inexpensive, small, lowpower, reliable, durable boxes. On one side they plug into the users' peripherals (such as the keyboard, monitor and mouse. On the other side, they connect via cables to the shared PC' (http://www.ncomputing.com/Solutions/howitworks.aspx).

So, in say a 30 desk classroom, just one computer can serve all 30 children, each of whom receives an access device, a monitor, keyboard, and mouse. There is no functional difference between this situation and one where each child is given a standard PC (as would be the case with a regular PC). Having one rather than 30 computers to install and maintain also represents a cost advantage for the shared option.

A major distinction between the two approaches is thus that the methods of access that they embody are diametrically opposite (one allows of no sharing and the other is based entirely on sharing). To gain insight into the appropriateness of these characteristics I turn next to a historical framework which shows the stylized changes in products as countries go from being poor to being relatively rich. First though I need to explain the concept of goods as embodied characteristics.

\section{The Analytical Framework}

\subsection{Goods as Embodied Characteristics}

In traditional demand theory preferences are defined over products. If a product is chosen the consumer is said to have revealed a preference for it. Products cannot be compared directly and it makes little sense to talk of distributional gains. In the newer approach by contrast products are viewed as bundles of characteristics over which individual and collective preferences are defined. The distributional impact of products then depends on the matching between preferences and characteristics. At the one extreme will be cases where a small group of high-income individuals will gain at the expense of the low-income majority whereas at the opposite extreme a wider spread among low-income beneficiaries is achieved. The basic model is set out in Fig. 1.

Each of the two goods, $\mathrm{X}$ and $\mathrm{Y}$, is represented by a ray from the origin. How far along each ray one can go depends on the price of the good and the budget. As demonstrated in this figure, for illustrative purposes, the consumer can reach A on good X and B on good $\mathrm{Y}$. Which of the two he will choose depends on his preferences for the two characteristics.

The person that favours characteristic 1 (as represented by the shape of the indifference curve that goes through $\mathrm{A}$ ) will choose product $\mathrm{X}$ and the person with opposite preferences will choose product $\mathrm{Y}$. Assume for example that characteristic 1 represents functional features of a product and characteristic 2 more luxury-type features, then a low-income person may tend to favour product $\mathrm{X}$ while the opposite is true for a relatively high-income person. Imagine that $\mathrm{X}$ is a simple bar of soap without any packaging and $\mathrm{Y}$ is a detergent with expensive packaging and other 'luxury' features such as optical whitening. 


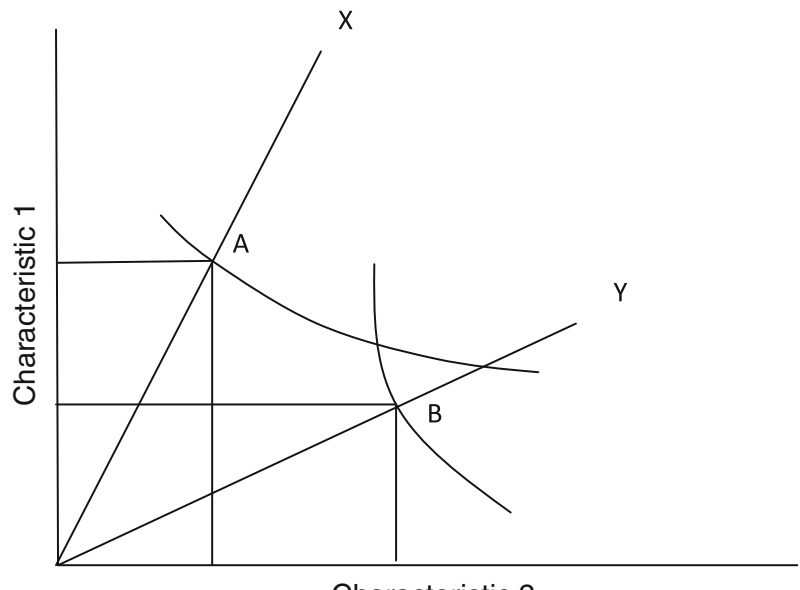

Characteristic 2

Fig. 1 The basic model

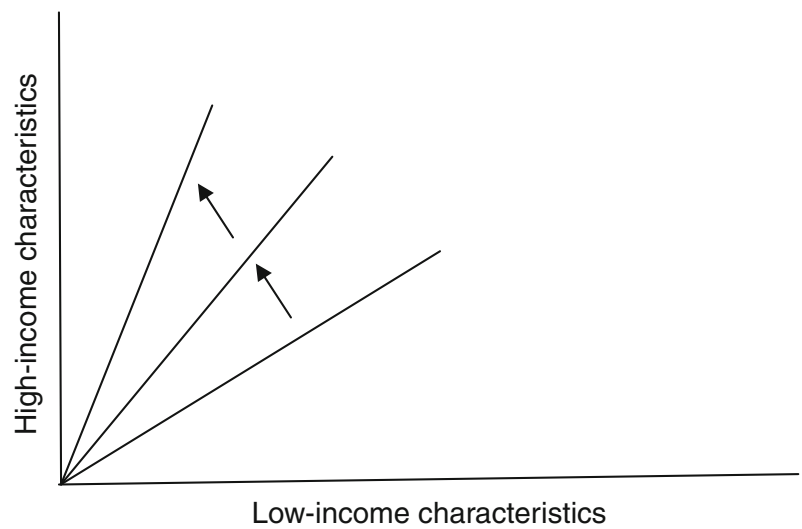

Fig. 2 Changes in product characteristics over time, Source: Stewart (1997)

\subsection{Product Characteristics and Income}

An important part of my framework is the recognition that there is a close relationship between income and the nature of product characteristics. More specifically as shown in Fig. 2 there is a tendency for products to become more 'high-income' over time.

As (Stewart 1997, p. 17) puts it:

Technical change in products in developed countries occurs in line with the rising incomes (and is partly responsible for those rising incomes), so that the balance of characteristics offered by new products corresponds to the changing demands of consumers as their incomes rise. The rising incomes have the effect of shifting demand towards different products with more sophisticated, labour-saving, higherquality, etc. characteristics: to summarize this complex of changes we may say that the characteristics of the new products have more high-income characteristics. 

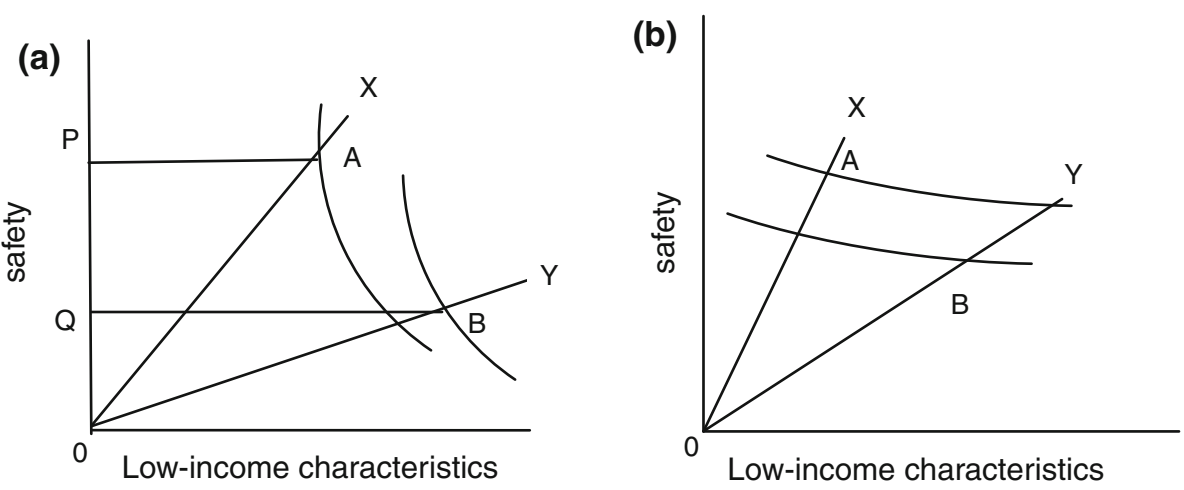

Fig. 3 The distributional impact of public goods

One common feature of new products for example is that they save time compared to older products as incomes rise. Take the case of cooking. In developing countries this is generally undertaken on a time-intensive basis but with rising incomes the balance of characteristics changes. Time becomes more scarce as wages rise and goods become more time intensive, through the use of capital-intensive modes of cooking (e.g. with coffee machines and micro-wave ovens). Note that this tendency is true not only of the physical properties of goods but also the mode of access to them, as will shortly become evident.

\subsection{Product Characteristics and the Distribution of Well-Being}

'In the case of public policy', writes Stewart (1997)

particularly public goods, there is a direct connection between the nature of the product produced and the distribution of income. The provision of particular types of public good itself affects the distribution of income. Given a limit in total public expenditure the allocation of funds to high-income inappropriate products automatically removes resources from low income consumers. For example, the provision of tarmacked highroads is at the expense of improving roads for the rural areas.

Consider from this point of view the situation depicted in Fig. 3. On the vertical axis is the characteristic safety, the standards of which in the developed countries reflect in a rough way the increased premium on this characteristic as incomes have grown (e.g. the increased safety of cars, airplanes and medical drugs). Assume in panel (a) of Fig. 3 that $\mathrm{OP}$ is the amount of safety associated with the developed-country product $\mathrm{X}$ (assumed to equal the minimum standard required), ${ }^{3}$ The local product $\mathrm{Y}$, by contrast is intensive in low-income characteristics and yields only OQ units of safety which is below the minimum required (OP). The result is that the average consumer in poor countries would be on a lower indifference curve because he or she is denied the chance to buy the cheaper, albeit less safe product that better accords with these average preferences. As shown in panel (b) however the category of inhabitants that favours safety in relation to low-income characteristics would benefit from the newer good X imported by the developing country. The outcome is that a minority of individuals with high-income preferences gains at the

\footnotetext{
${ }^{3}$ I have made the developed country product exactly meet the standard. The general point is that it is more difficult to meet the standard with developing as opposed to developed country products.
} 
expense of the majority. From a distributional point of view this can only described as unequal.

As another example of the situation in Fig. 3 consider healthcare and in particular manually operated X-Ray equipment which is no longer approved for use in developed countries because it is considered unsafe. This type of equipment has been replaced by more complex and safer machines. In developing countries, however, it is tempting to assume that 'the benefits of having X-Ray equipment available for use outweighs the potential risk of unnecessary exposure from manual operation' (Kennedy 1979). Or again expenditure of the government health budget may take the form of relatively few high-cost, low-risk products from developed countries or cheaper, older and more risky alternatives. The latter option would permit a much higher percentage of the population in developing countries to get at least some drug therapy. And it would accordingly represent a much more equal distribution of well-being.

Finally, I turn to the field of housing where developing country governments are notorious for clinging to irrelevant developed-country standards, which the majority of the population cannot afford, 'Purchase of the housing with 'too-high' standards involves a disproportionate expenditure on this item compared to other items in the household budget. i.e. it leads to imbalances in consumption (or to concentration of public expenditure on housing to the exclusion of other social needs) '. ${ }^{4}$ For example, in a slum upgrading project in Kibera, Kenya, 'One cannot blame Kibera residents for assuming that the project is deliberately attempting to create housing for the middleclass and deprive current-residents of their right to a convenient location in Nairobi' (Huchzermeyer 2006, p. 18).

In the next and final element of the analytical framework I focus specifically on the two characteristics that seem best to discriminate between the alternative models of computing described above.

\subsection{Individual Access as a High-Income Characteristic (and Sharing as a Low-Income Characteristic)}

Generally, the relationship between per capita income and individual access (as a percentage of population) looks something like the line $0 \mathrm{Z}$ portrayed in Fig. 4.

In developed countries (at say Y2) most people are easily able to afford the cost of owning a computer. At developing country levels of income (Y1) this is also true of the upper-income deciles of the population. But for the relatively poor majority in these countries the main alternative mode of access to computers is sharing. Indeed numerous authors, across different developing regions, speak of a culture of sharing. For example, 'the Philippines have a culture in which household members generally share resources' (Rangaswamy and Singh 2009). In Asia more generally, 'material resources are often shared at the level of household and neighborhood.....Asian cultures privilege the family over the individual' (Rangaswamy and Singh 2009). Or again in a different region and in relation to the mobile phone 'in some African countries it is regarded as the property of the community, because there is a culture of sharing the tools of communication' (Lopez 2000).

James (2010a) argues that the amount of computer sharing in developing countries should indeed be greater than in developed countries, to an extent that depends on the income differences between them. The frame of reference is a developed country, the UK, which at a per capita income level of $\$ 42,740$ has 5 students per computer (this is the actual

${ }^{4}$ See James (1982, p. 264). 
Fig. 4 Incomes and individual access

Table 1 Appropriate levels of sharing, selected countries

GNI gross national income, $O L P C$ one laptop per child

Source: James (2010a)

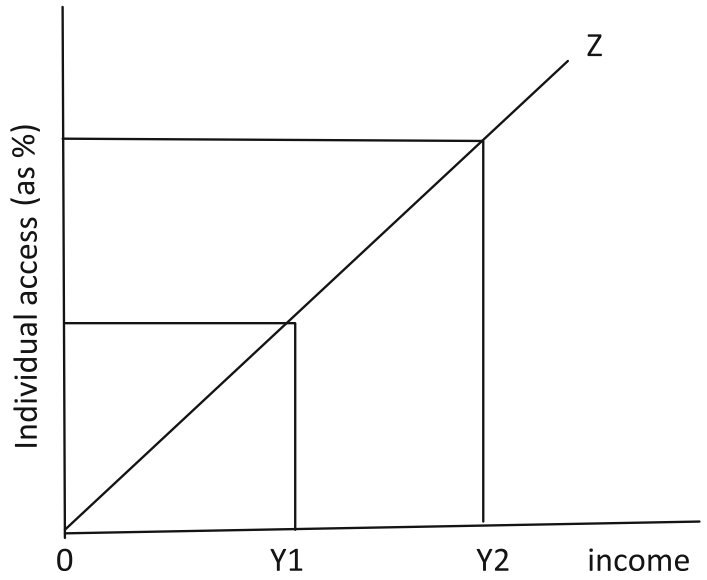

\begin{tabular}{llll}
\hline & $\begin{array}{l}\text { Per capita GNI } \\
\text { (atlas method) in } \\
\text { current dollars }\end{array}$ & $\begin{array}{l}\text { Ratio of GNI } \\
\text { per capita to } \\
\text { OLPC (\%) }\end{array}$ & $\begin{array}{l}\text { Number of } \\
\text { students per } \\
\text { one laptop }\end{array}$ \\
\hline OLPC & 213,700 & 100 & 1 \\
United Kingdom & 42,740 & 20 & 5 \\
Kenya & 680 & 0.32 & 312.5 \\
Low-middle income countries & & \\
Thailand & 3,400 & 1.6 & 62.5 \\
Peru & 7,958 & 3.7 & 27 \\
India & 950 & 0.44 & 227.3 \\
Philippines & 1,620 & 0.76 & 131.6 \\
China & 2,360 & 1.1 & 90.9 \\
Colombia & 3,120 & 1.45 & 69 \\
Dominican republic & 2,038 & 0.95 & 105.3 \\
\hline
\end{tabular}

situation there and also the level recommended by education authorities in other developed countries such as the USA). To arrive at the 1:1 (Rangaswamy and Singh 2009) relationship proposed by the OLPC, the British income has to be multiplied by 5 , yielding a figure of over $\$ 200,000$ (see Table 1). In effect the OLPC is basing its solution on an income level that no country on earth comes close to attaining. Take the case of Kenya shown in Table 1. The recommended amount of students per laptop of 312.5 is reached by calibrating the per capita income of Kenya (\$680) against \$213,700, 'In particular, the ratio of the former to the latter level of gross national income (GNI) per capita is equal to $0.32 \%$ which, in the ratio of 100:32, gives the balanced amount of computer spending per child (in this case, 1 computer should be shared by 313 Kenyan students)'. (James 2010a). For more developed countries such as Peru, however, the amount of sharing drops to 27 students per computer. With the ' $\mathrm{N}$ Computing' model this amount is easily accommodated whereas the OLPC solution would require a per capita income that is 29 times the figure of Peru (i.e. $\$ 213,700)$. At lower levels of income the purchase of 'XO' computers for all students, would result in serious imbalances in the education budget and even in other sectors. This is the inevitable consequence of applying (higher than) developed country standards to 
poor countries (or relying on a decidedly high-income characteristic to solve a poor country problem).

\section{Application}

So far I have suggested that 'N Computing' is a product that efficiently promotes sharing (a low-income characteristic) whereas the OLPC program entirely excludes this mode of access to a computer. This difference, I further suggest, is the primary reason why the former is associated with a much lower cost per pupil than the latter. The details are shown in Fig. 5 where the two products coincide with the axes, corresponding to the case of nosharing and total sharing. ' $\mathrm{N}$ Computing' has a per pupil cost of about $\$ 70$ which is two and a half times lower than the 'X0' computer (selling as it does at around \$180). A given expenditure that is to say would result in two and a half times more students with computer access if it was invested in the one case rather than the other. The distributional impact is clearly greater since it reaches many more pupils (there is no information however on the income level of those who benefit from this product as opposed to those who do not).

It would be wrong though to consider the ' $\mathrm{X} 0$ ' laptop as an entirely inappropriate product. After all, it was designed specifically to meet the needs of people living in poor rather than rich countries. Because of the many innovations that were generated with this goal in mind the ' $\mathrm{X} 0$ ' is able to be sold at a price that is well below that of the typical computer in developed countries.

In this respect the 'OLPC' computer can properly be described as appropriate (as also can Intel's low-cost computer, 'The Classmate'). What makes the 'OLPC' computer inappropriate, however, is it's insistence on using the same costly high-income mode of access that is used in rich countries. In developing countries sharing is a more appropriate characteristic because it allows many pupils to use the available resources of a single computer. These are resources moreover, that would otherwise go unused, especially in developing countries where computers tend to rely on an especially small proportion of

Fig. 5 A comparison of ' $\mathrm{N}$ Computing' and the 'OLPC'

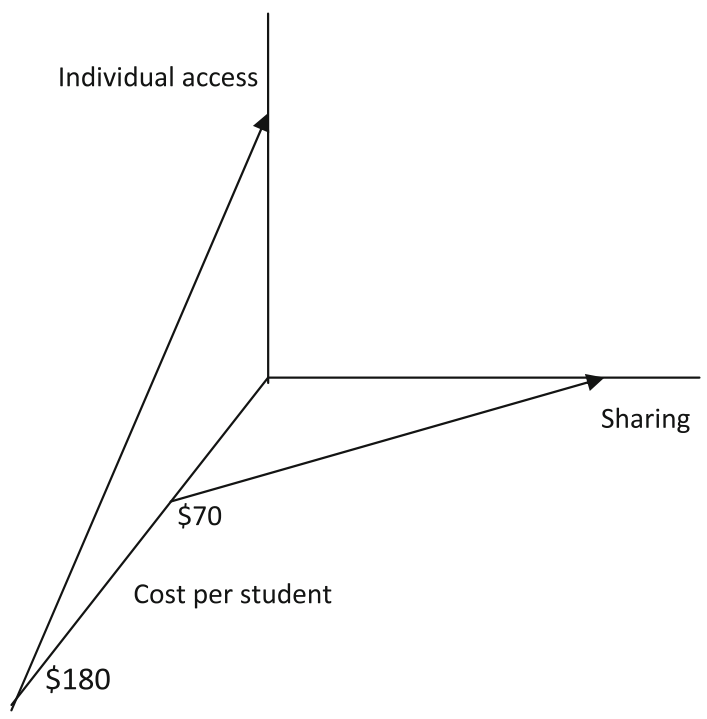


their full capacities (partly because of a lack of skills). ${ }^{5}$ Given the cost differences between the two computers shown in Fig. 5 it seems evident that the appropriate aspect of the OLPC product is outweighed by its reliance on a clearly inappropriate characteristic.

It is worth emphasizing here how much desktop virtualization improves on traditional methods of sharing (after all, much of what appropriate technology is about, is upgrading traditional practices). To illustrate this point consider a class size of 30 pupils and 1 computer. In traditional sharing, the pupils would have to take turns in using the computer. This option however runs the risk that users will not get sufficient time per week to learn the basic computing skills (1 hour per week for example is thought by some to be insufficient for this purpose). The ' $\mathrm{N}$ Computing' model avoids this problem because it provides 30 times more hours for sharing, since 30 virtual computers can be connected to the original one as described above. (For the sake of completeness, note that the OLPC computer would be given to all 30 students).

Is also bears emphasis that the 30 students will increase their skills as they make the transition to higher educational levels. This means that more than one computer will be required in addition to the relevant number of access devices, monitors and so on.

\section{The OLPC Response}

Faced with the cost data presented in Fig. 5, proponents of the OLPC are apt to argue that their product is different from and superior to what ' $\mathrm{N}$ Computing '(and many other lowcost computer manufacturers) make available. The ' $\mathrm{X} 0$ ' for example can be taken home and shared with the family (who might then also benefit). The OLPC computer is also said to encourage pupils to become more engaged with and involved in their own education. (In a way that is predicted by Papert and others). ${ }^{6}$

Although these issues are still being debated, let us assume for the sake of argument that the ' $\mathrm{X} 0$ ' is indeed a superior product in the respects that have just been mentioned. This assumption makes for a starker comparison between the two distributional outcomes that were described above. For, what one is then confronted with is a situation in which relatively few pupils enjoy a superior education and one where more pupils get a basic education. (It thus represents a more equal distribution of computer use in the sense defined above.) OLPC proponents, however, have yet to make a case why one should prefer the former over the latter (more equal) outcome. Without this argument countries in the Third World may well be apt to choose the ' $\mathrm{N}$ Computing' alternative as India has recently done. ${ }^{7,8}$

\section{Conclusions}

So far neither the literature on appropriate technology or appropriate products has incorporated sharing as a relevant characteristic. Yet, as I have tried to show, this characteristic

\footnotetext{
5 See Cawthera (2003).

${ }^{6}$ Papert is associated with a theory of learning called constructionism.

7 Another well-known case is Macedonia which deployed 180.000 student seats, complete with 15-inch screens, shared computers and all software and peripherals for less than $25 \%$ of the price of the cheapest computer per seat deployment.

8 Thus far, OLPC's argument in favour of one child per computer is not at all convincing. One of the arguments for example is that just as people own toothbrushes so too should they own computers.
} 
is central to the comparison of two of the leading low-cost computers now available to developing countries. Sharing emerged in particular as a low-income feature of the product sold by ' $\mathrm{N}$ Computing' that helps to foster a relatively equal distribution of computer use (by allowing more children to have simultaneous access to computers). The OLPC product by contrast allows of no sharing-relying instead on the high-income characteristic of individual access- and in spite of having some low-income characteristics generates a more unequal distribution of computer use. Proponents of this product have to convince governments why it is better to give an arguably superior educational experience to relatively few pupils as opposed to providing far more students with a basic education in countries where access to computers in schools is highly limited. ${ }^{9}$ Without such an argument developing counties may be apt to favour the sharing-intensive product as India has recently done.

In the future, however, things may change if the Indian \$35 laptop materializes. At present it is only at the prototype stage and many observers are skeptical that it will actually sell at that price if it emerges at all. After all, experience has shown that price forecasts at the prototype phase-in India and elsewhere-have often proved to be overoptimistic.

Open Access This article is distributed under the terms of the Creative Commons Attribution Noncommercial License which permits any noncommercial use, distribution, and reproduction in any medium, provided the original author(s) and source are credited.

\section{References}

Cawthera, A. (2003). Computers in secondary schools in developing countries: Costs and other issues. Washington D.C: The World Bank.

Greenberg, A. (2008). One Virtual PC per Child, Forbes 13 October.

Huchzermeyer, M. (2006). Slum upgrading initiatives in Kenya within the basic services and wider housing market, Discussion paper No. 1, Kenya Housing Rights Project, COHRE Africa Programme.

James, J. (1982). Product standards in developing countries. In F. Stewart \& J. James (Eds.), The economics of new technology in developing countries, Pinter.

James, J. (2010a). New technology in developing countries: A critique of the one-laptop-per-child program. Social Science Computer Review, forthcoming.

James, J. (2010b). Sharing mobile phones in developing countries: Implications for the digital divide, mimeo.

Kennedy, D. (1979). Food and drug administration and pharmaceuticals for developing countries, in US National Academy of Sciences, Pharmaceuticals for Developing Countries, Washington D.C.

Lopez, A. (2000). The South goes mobile. The Courier. UNESCO.

Rangaswamy, N., \& Singh, S. (2009). Personalizing the shared mobile phones, HCI (vol. 14).

Stewart, F. (1997). Technology and underdevelopment. London: Macmillan.

\footnotetext{
${ }^{9}$ I have dealt with education in this paper, but the sharing model can also be applied to the village or regional levels in the form of so-called telecentres. These are almost always financed by foreign aid though the local community can be asked to pay something on a per-use basis. Here, too one might expect a more equal use of shared computers compared with regular PCs.
} 